International J. of Health Services Research and Policy (2019) 4(1): 31-42

\begin{tabular}{|l|l|}
\hline \multicolumn{1}{|l}{$\begin{array}{l}\text { INTERNATIONAL } \\
\text { ENGINEERING, } \\
\text { SCIENCE AND } \\
\text { EDUCATION } \\
\text { GROUP }\end{array}$} & $\begin{array}{l}\text { International Journal of Health Services Research and Policy } \\
(2019) \text { 4(1):31-42 }\end{array}$ \\
& Published online April 2019 (http://dergipark.gov.tr/ijhsrp) \\
& doi: 10.23884 ijhsrp.2019.4.1.04 \\
& e-ISSN:2602-3482 \\
& Received: January 5, 2019 Accepted: March 12, 2019 \\
& Submission Type: Research Article \\
\hline
\end{tabular}

\title{
ASSESSING INSTITUTIONAL CAPACITY OF COUNTY HEALTH DEPARTMENTS IN KENYA USING THE HEALTH SYSTEMS FRAMEWORK: IMPLICATIONS FOR SERVICE DELIVERY AND OUTCOMES \\ Vincent R. OKUNGU ${ }^{1}$
}

ORCID: 0000-0002-1359-2645

${ }^{1}$ School of Public Health, University of Nairobi, Nairobi, Kenya

*Corresponding author:okungu008@gmail.com

\begin{abstract}
The study used the county institutional capacity assessment tool (CICAT) to assess the capacities of selected county health departments (CHDs) along the World Health Organization (WHO) health systems framework that is organized around the six building blocks. The aim was to determine the current status of the six health system building blocks in Kenya's county health departments. The article emanates from data collected for a technical report on county institutional capacity assessment. Data were analysed using Microsoft Excel spreadsheet and thematic analysis. The overall performance of the counties was 54\% indicating average capacity. The performance scores were: leadership and governance (39\%), human resources for health (44\%), health information systems (66\%), medical products and technologies (50\%), health system financing (50\%), and service delivery (71\%). There was no statistically significant difference in the overall performance of the counties $(p=0.892)$. The key issues in some of the worst performing building blocks included poor work-planning and lack of transparency in budgeting and expenditure, low funding and irregular disbursements of health budgets, lack of key policies, e.g. workforce strategy and commodity supply chain. In conclusion, the study proved that the CICAT is useful for assessing the process of strengthening health systems in Kenyan counties.
\end{abstract}

Key Words: Capacity development, health department, county, Kenya

\section{Introduction}

Capacity development is essential for institutional ownership of policies and plans; for institutional efficiency; for community empowerment; and for maintaining nationwide progress over time [1]. Capacity is defined by the Organization for Economic Co-operation and Development's- Development Assistance Committee (OECD-DAC) as the "the ability of people, organizations, and society as a whole to manage their affairs successfully; and capacity development as the process by which people, organizations and society as a whole create, strengthen and maintain their capacity over time"[2]. As opposed to the traditional view of capacity development as all about human resource development, the broader scope includes whole system and institutional transformation and local ownership, policy impact, and sustainability[1].

Capacity development in the health sector is crucial to the realization of national health agenda and the Sustainable Development Goals (SDGs), particularly SDG 3 - Ensure healthy lives and promote well-being 
for all at all ages. To achieve health sector goals, Kenya devolved its governance system in 2013 and created devolved units of governance called counties. County governments are largely responsible for service delivery through respective county health departments (CHD). To strengthen service delivery at county level, the national government has partnered with development agencies to assess and develop capacities of CHDs across the country. To this end the United States Agency for International Development (USAID) developed a county institutional capacity assessment tool (CICAT) to guide assessment of capacities and formulation of capacity development strategies for CHDs. The WHO health systems framework consisting of six building blocks was used to guide the capacity assessment of the CHDs.

The WHO [3] explains the six building blocks as follows: Leadership and governance involve ensuring the existence of policy frameworks combined with effective oversight, coalition building, regulation, attention to system design and accountability. A well-performing health workforce is responsive to patient needs, fair and efficient to achieve the best health outcomes possible under prevailing financial and social conditions. A well-functioning health information system ensures the production, analysis, dissemination and use of reliable and timely information on health determinants, health system performance and health status. A well-functioning health system ensures equitable access to essential medical products, vaccines and technologies of assured quality, safety, efficacy and cost-effectiveness, with efficiency in use. A good health financing system raises adequate funds for health, in ways that ensure people can use needed services when they need them and are protected from financial catastrophe or impoverishment. Service delivery is an immediate output of the inputs into the health system, such as the health workforce, procurement and supplies, and financing. Services delivered are expected to be of sufficient quality to be effective, are available to those who need them when they need them $[4,5]$.

Effective and sustainable capacity development requires institutionalized capacity needs assessment to progressively identify and address capacity gaps. For example, CHDs will need to improve in all the six health system building blocks and coordinating them in ways that ensure equitable and sustainable health benefits and outcomes across diverse population groups. This requires technical and political knowledge and action [6]. The assessment is particularly critical for the counties which are responsible for service delivery and the country as a whole as it makes reforms for universal health coverage (UHC).

The goal of the assessment was to develop a shared understanding of the current capacity of the institutions and organizations that the management of the CHDs represent in order to analyse gaps and develop a responsive capacity building strategy in the form of action plans. USAID Health, Population and Nutrition (HPN) planned to use assessment results to develop leadership, knowledge and skills of county health department officials; and strengthen their planning, performance, oversight and public financial management and revenue generation systems.

\section{Methods}

The manuscript comes from analysis of data from an institutional capacity assessment exercise conducted by USAID and International Business and Technical Consultants Inc. (IBTCI). The study was a cross-sectional survey. The data were collected using a questionnaire tool that was adapted from and harmonized with a number of Organizational Capacity Assessment Tools (OCAT). Data were collected from February to June 2018 in five purposively selected administrative zones (counties): Busia, Kakamega and Migori counties to the west of Kenya, Mombasa County at the coastal region, and Turkana County to 
the north. The five are part of USAID priority counties in Kenya and were selected based on USAID's current health portfolio investments [HIV/AIDS, reproductive, maternal, neonatal, child \& adolescent health (RMNCAH), nutrition and malaria], and presence of other development partners for effective leveraging of resources for greater impact.

Questionnaire administration was collaborative; i.e. it was first and foremost a self-assessment tool, meaning that study participants including members of the county and sub-county health management teams (CHMT) and (SCHMT), and implementing partners worked through each component of the tool together. The total number of participants per county in the assessment ranged from $20-24$. The results of the assessment for each county were later validated in a workshop held in each county. The total number participating in the validation workshops ranged from $34-38$ including the original participants in the initial assessment exercise.

The questionnaire included a summary score sheet organized by building block. Scores were weighted from 0 to 4, being the lowest and highest scores, respectively. Zero meant no indication of capacity; a score of 1 meant only the very basic indicators of capacity existed; 2 meant that indicators in 1 in addition to another or others; 3 meant existence of all indicators in 2 in addition to another/others, and 4 meant high capacity involving all the indicators in 3 plus other attribute(s). A 4-point Likert scale (Table 1) was used to rank indicators of capacity for each building block using defined attributes/standards (see tool summary in Annex 1), while a 5-point Likert scale (Table 1), expressed in percentages, was used to provide overall capacity indicator by county.

Table 1. Indicator standards score: 4-point Likert Scale and Score Matrix for the CICAT

\begin{tabular}{|l|l|}
\hline $0-1$ & No Capacity \\
\hline 2 & Low Capacity \\
\hline 3 & Moderate Capacity \\
\hline 4 & High Functional Capacity \\
\hline Overall indicator Score: 5 -point Likert Scale \\
\hline $20 \%$ \& below & No Capacity \\
\hline $21 \%-39 \%$ & Limited Capacity \\
\hline $40 \%-59 \%$ & Average Capacity \\
\hline $60 \%-79 \%$ & Significant Functional Capacity \\
\hline $80 \% \&$ above & Very Significant Functional Capacity \\
\hline
\end{tabular}

$\mathrm{NB} /$ All scores were out pf 16 (100\%) except service delivery which was scored out of 20 (100\%)

The WHO's six building blocks served as the analytical domains. Basic descriptive statistics were used in the analysis. A trend analysis was used to determine the overall change in key health financing indicators over the last 4 years since the start of devolution, plotting year by year to assess the level of the quantitative indicators using basic statistical analysis methods. For the qualitative component of the evaluation study, content analysis was used to identify key themes and categories to support quantitative evidence generated from the questionnaire.

\section{Ethical approval}

Secondary data sources were used in the manuscript development so no ethical approval was required. 


\section{Results}

\subsection{Overall performance by county}

Figure 1 illustrates total capacity of counties before and after validation of the results of the assessment. The scores after validation were taken as the final scores of the evaluation study.

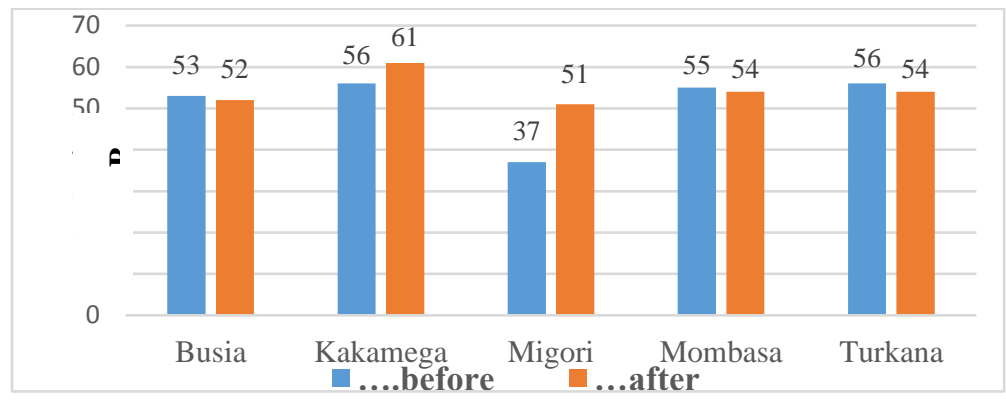

Figure 1. Capacity scores before and after validation of findings

There were no major differences in the scores before and after validation of findings except in Migori County, which was explained on inability to locate key documents before validation of the findings. Oneway ANOVA returned no statistical difference in the overall performance of the five counties $(\mathrm{P}=0.892)$.

\subsection{County capacity scores by building block}

This section shows total average scores per county by building block. The total average score in all the six building blocks was $54.3 \%$ indicating average capacity for all the counties combined but with individual county variations. On individual building blocks (Figure 2), the total average capacity in leadership and governance block (39\%) is the lowest with Mombasa County showing the greatest weakness in this block. As a key building block, it suggests a fundamental weakness in the health system even as Kenya makes reforms toward UHC. Health information systems (HIS) block is the best performing with all the counties demonstrating ( $66 \%$ on average).

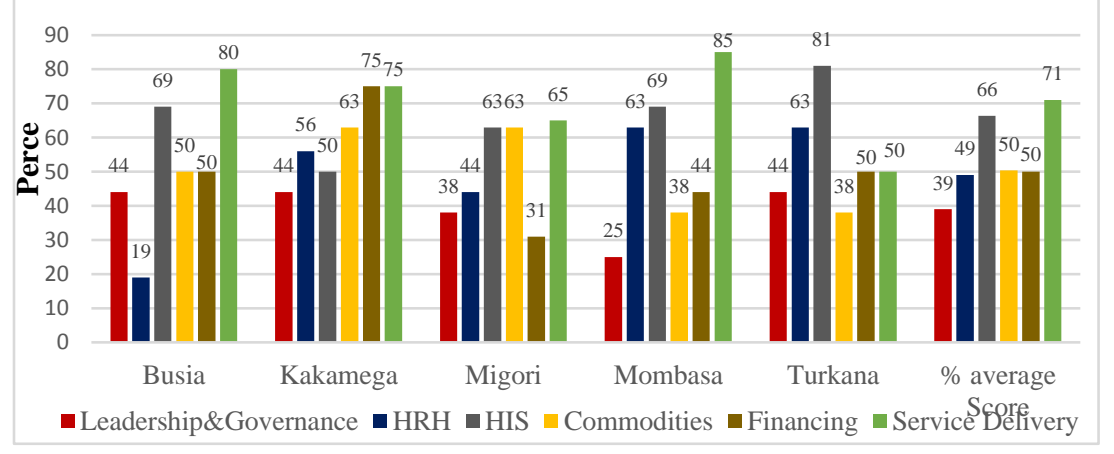

Figure 2. Total capacity of counties in each Building Block

[KEY: $20 \%$ \& below= No capacity; $21 \%-39 \%=$ Limited capacity; $40 \%-59 \%=$ Average capacity; $60 \%-79 \%=$ Significant functional capacity; $80 \%$ \& above $=$ Very significant functional capacity]

There is average capacity (50\%) in the health commodities and technologies block. Qualitative data indicated persistent transport problems as the main issue affecting distribution of commodities in health facilities in low performing counties such as Turkana and a lack of supply chain data tracker in Mombasa CHD. In the Financing block, Migori County due to low transparency in budget allocation and rigid control of health funds by the county treasury. 
A trend analysis of health financing for all the counties (Figure 3) indicated limited yet unpredictable financing for most of the counties, which hinders planning and delivery in priority services. The trends suggest relatively high budget allocations by county governments to the county health departments, e.g. $30.1 \%$ of total county budget in Kakamega County. However, in terms of per capita spending, the figures are very low ranging from USD 15.2 (Migori) to USD 21.3 (Mombasa). Even with input from the national government, such levels of expenditure do not meet the USD 90 recommended by the WHO as the minimum per capita health spending required to guarantee a minimum package of health services.

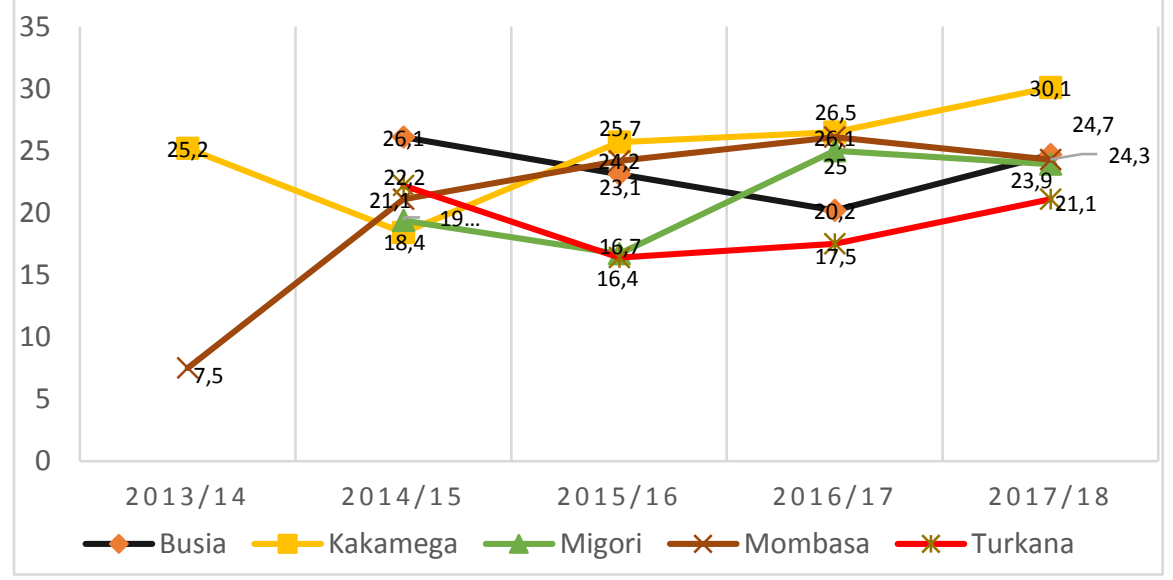

Figure 3. Health budget allocations as a percentage of total county budget

The Service Delivery block is quite strong at $71 \%$ on average (significant functional capacity). The high scores could be attributed partly to the technical and financial engagement by donor agencies which largely support services related to HIV/AIDS, malaria, TB, and reproductive, maternal, neonatal, and child health $(\mathrm{MNCH})$ services.

\subsection{Factors driving county performance in each building block}

The results from the indicator scores are summarized in Figure 4.

[Key: $0-1=$ no capacity; $2=$ low capacity; $3=$ moderate capacity; 4= high functional capacity]

From the indicator scores, in the Leadership and Governance (L\&G) block, all the counties except Turkana have significant capacity to develop and implement a county health strategy. Lack of funds and technical capacity were the main reasons cited for the lack of capacity to develop and implement a county health department strategic plan in Turkana County. The main weakness for all the five counties was in their "capacity to communicate effectively within the county and sub-county health department and other departments within the county". From the qualitative data, this was explained on absence of a communication strategy and key protocols guiding information flow within the CHD and between the CHD and implementation partners. Turkana and Migori counties demonstrate significant capacity to "lead and engage with different health actors working towards a common goal", a factor which was explained on a clearly defined memoranda of understanding (MOUs) with implementing partners. Overall, Mombasa CHD demonstrated 'no capacity' in most indicators which was partly linked to a lack of clear policy guidelines on health sector priorities as well as irregular update meetings to discuss emerging leadership challenges at county, sub-county and community levels. 
International J. of Health Services Research and Policy (2019) 4(1): 31-42

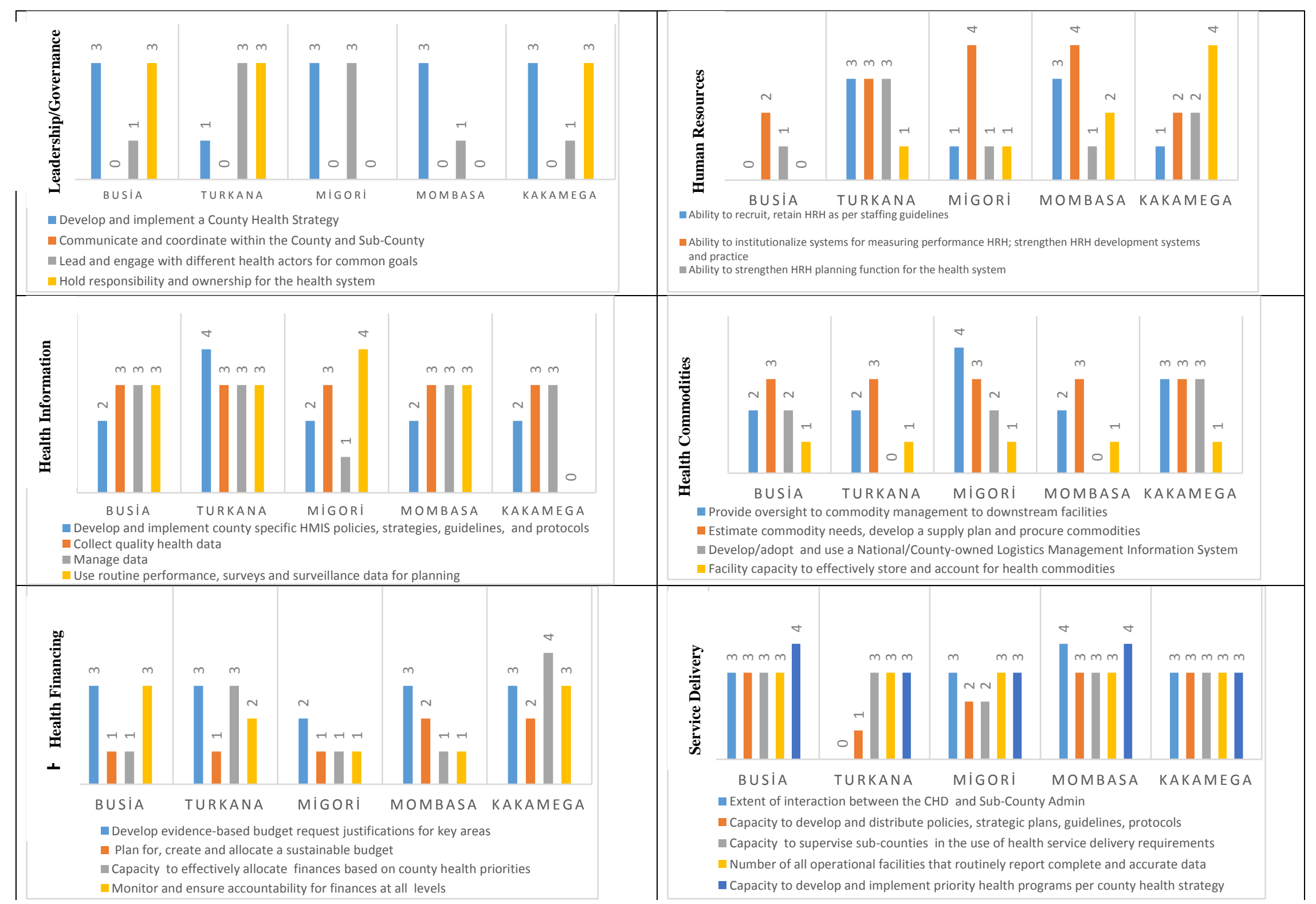

Figure 4. Indicator scores for each Building Block by county 
On the whole, the most outstanding issues contributing to the general weakness in L\&G block for all the counties included lack of a monitoring and evaluation framework to track the progress of county health sector strategic and investment plan (CHSSIP) as well as inadequate capacity to develop work plans which affects definition of health department goals and objectives. Besides, the governance structure fails to properly define the roles and responsibilities of key actors in achieving county health strategy goals. The actors are poorly coordinated which affects their involvement in annual sector reviews, work plan development and policy formulation.

In the human resources (HRH) block, the significant capacity showed particularly by Turkana County, a remote and hardship area, was linked to the political leadership in the county that is keen to attract and retain health workers. The county health department has since rolled out a number of incentives including housing, hardship allowances, transport and annual leave allowances as well as training opportunities, to attract and retain health workers. Busia and Migori CHDs have no capacity in all but one component of HRH. The main challenges in both counties include a lack of strategy to attract, recruit and retain $\mathrm{HRH}$, lack of incentives as well as poor working conditions including lack of opportunities for feedback, unavailability of commodities at health facilities, poor dissemination of HR policies and lack staff needs assessment.

The health information systems (HIS) block is performing well; however, there are still capacity gaps affecting all the counties. These included inadequate regular data analysis and sharing with key actors such as CHMTs, SCHMTs and non-state actors for use as evidence in strategic planning and policy making including rational budgeting and decision-making; inadequate supply of data collection tools especially at facility and community levels, which affects timely forecasting for commodities and overall quality of services. Other capacity gaps include inadequate staff capacity on data management (knowledge and skills) as well as poor dissemination of M\&E framework, plans, protocols and guidelines to the sub counties.

In the health commodities and technologies block, there are serious weaknesses in specifically two areas: capacity to develop and/or adopt and use a national/county-owned logistics management information system (LMIS), and capacity for facilities to effectively store and account for health commodities. The main focus for all the counties in this building block should be to build capacity to effectively store and account for all health commodities alongside a fully functional LMIS. The key issues reported in all the counties included lack of data quality improvement plan for all LMIS elements as well as lack of adequate storage for health commodities including special storage needs at all levels (county, sub-county and health facility).

Health system financing block is quite weak especially in Migori County where there is no capacity in all nearly all the indicators. Qualitative data suggested that Migori CHD specifically lack transparency in budgetary allocations as well as unclear policies in identifying and guiding the implementation of county health system priorities. From qualitative data, the main difference between Kakamega CHD, which performs best in this block, and the others, is the political will that has prioritized the health sector and has fostered transparent and accountable budget systems that target evidence-based county health priorities. In as much as not all budgetary wishes are granted, the health sector as a whole faces serious under-funding, which is complicated by a lack of department expenditure tracking and quarterly review framework. This often makes the county health department funds exposed to misuse through corruption and other forms of fraud. Other key weaknesses as informed by the qualitative data included inadequate use of evidence-based data to inform budget allocation, delays in disbursement of funds from the 
national and county treasuries, and lack of skills in programme-based budgeting (PBB) especially at sub-county level which affects proper linkages between the budgets and work plans. The main issue with PBB implementation is lack of capacity to develop clear and implementable programme and subprogramme objectives and performance indicators. Although most counties have been trained on PBB, there were no follow ups to entrench PBB skills at county and sub-county levels.

In the Service Delivery block, all the counties are performing relatively well except Turkana County. The main reason for the lack of capacity in Turkana County was explained on insecurity and poor road network. This has hampered delivery of commodities to health facilities as well as discouraged potential development partners from working in the county. For all the counties, there is limited interaction between the county health department headquarters, the sub-counties and community units. This has affected effective planning and budgeting that takes into account input from different stakeholders. Other key weaknesses included irregular monitoring of compliance in the use of standards and guidelines at sub-county and health facility levels, and poor data management.

The service delivery portfolio was further disaggregated into specific services that are identified as key priority programmes for the counties: HIV/AIDS, malaria, TB/Leprosy, RMNCH, Nutrition, Water \& Sanitation, and NCDs (Figure 5).

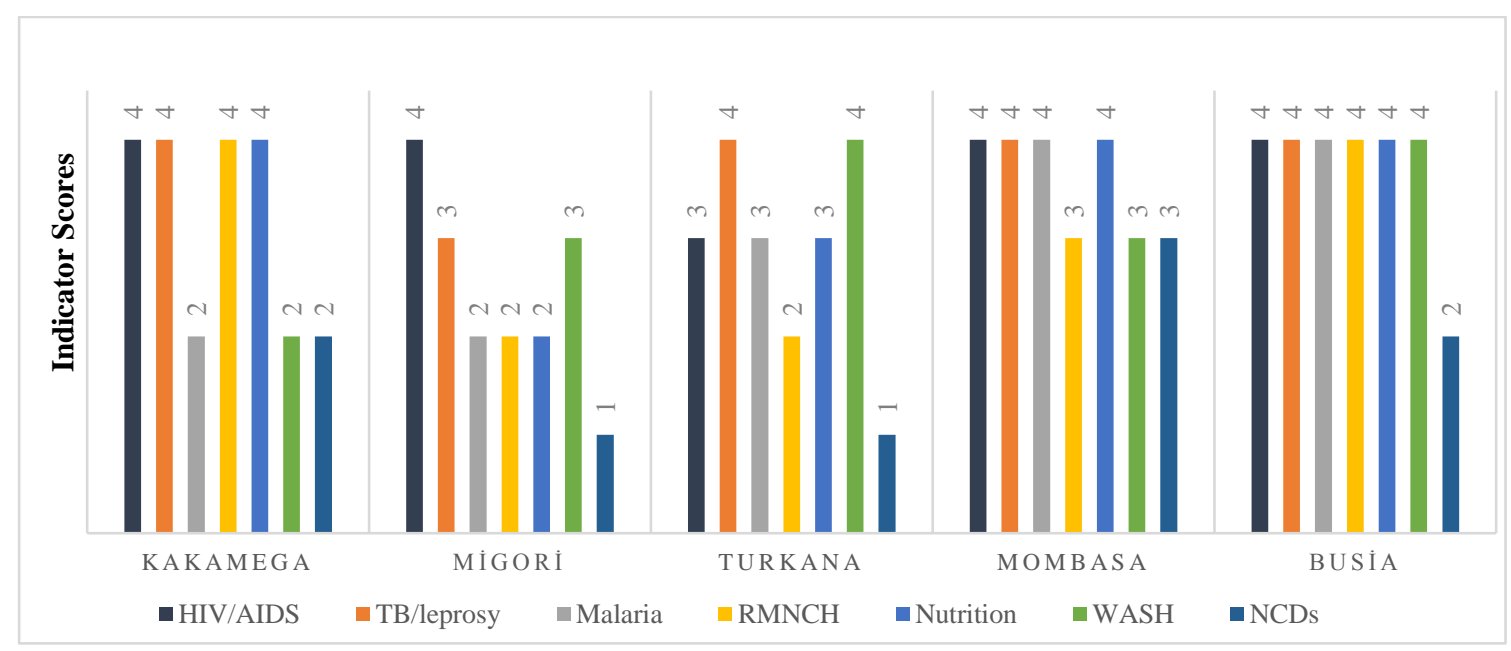

Figure 5: Capacity in specific priority service delivery areas

All the counties have very significant capacity in HIV/AIDS response largely due to the efforts of donor agencies but very low scores in NCD response. As an emerging health threat, NCDs have not received funding as a priority area over the years.

\section{Implications on health outcomes}

The health outcomes for the five counties are summarized in Table 2.

Table 2: Key population health indicators by county (Sources: [7-10])

\begin{tabular}{|l|l|l|l|l|l|l|}
\hline & $\begin{array}{l}\text { \% Immunization } \\
\text { coverage }\end{array}$ & $\begin{array}{l}\text { \% Facility } \\
\text { delivery }\end{array}$ & $\begin{array}{l}\text { MMR/ } \\
\mathbf{1 0 0 , 0 0 0}\end{array}$ & $\begin{array}{l}\text { U5-MR/ } \\
\mathbf{1 0 0 0}\end{array}$ & NMR/1000 & $\begin{array}{l}\text { (\%)HIV/AIDS } \\
\text { prevalence }\end{array}$ \\
\hline Kakamega & 81 & 69 & 316 & 64 & 19 & 4.0 \\
\hline Migori & 36 & 55 & 673 & 82 & 19 & 14.7 \\
\hline Turkana & 56 & 30.5 & 1594 & 74 & --- & 7.6 \\
\hline Mombasa & 64 & 82 & 223 & 32 & --- & 7.4 \\
\hline
\end{tabular}




\begin{tabular}{|l|l|l|l|l|l|l|}
\hline Busia & 80 & 58 & 307 & 11 & 24 & 6.7 \\
\hline $\begin{array}{l}\text { National } \\
\text { average }\end{array}$ & 67 & 61 & 362 & 52 & 22.6 & 6.0 \\
\hline
\end{tabular}

The results in Table 2 indicate that the worst performing county in service delivery (Migori) also has the worst health outcomes, which suggests a direct correlation between the progress in the building blocks and health outcomes. Although Busia County is not the best performing overall, it out-performs the rest of the counties in service delivery leading to better health outcomes including full immunization (80\%, utilization of ITNs $(80 \%)$, facility delivery $(58 \%)$, etc.

\section{Discussion and conclusion}

The study focused on USAID priority counties and the results may not fully reflect other counties, which have not experienced the same level of investment. Nevertheless, there are critical lessons learnt on the need to improve the performance of CHDs for effective service delivery. The study aimed at assessing the current status of CHDs along the six building blocks. In general, all the counties have average capacity and in terms of progress toward UHC, will have to make significant investment in all the building blocks to be successful. There are individual county variations in capacity and the key issues driving these variations include the political will that prioritizes health care, transparency in budget allocations to the health department, dissemination of key policy documents and protocols, and a communication strategy to help in coordinating both inter- and extra-county affairs. Getting these key areas right will contribute to strengthening all the building blocks.

Recently, an increasing number of international and local organizations have taken keen interest in health systems strengthening (HSS) as a basis for improved health for all [11-13]. The Kenya Government has also moved to strengthen the health system through such measures as devolving delivery of health services to the local units (counties) to ensure responsiveness to local needs; i.e. county governments are now entirely responsible for health system management and service delivery. This analysis helps devolved health systems to understand their key strengths and weaknesses to allow for targeted interventions and sustainable progress in HSS and UHC.

The findings indicate that lack of key health systems governance structures such as lack of a monitoring and evaluation framework and a communication strategy, contributes to significant weaknesses in the leadership and governance block; e.g. it makes it impossible to track progress of county health sector strategic and investment plans (CHSSIP). Furthermore, the incapacity to develop and/or implement annual work-plans at all levels of county departments of health affects implementation of quality health programmes. Work plans are constructed within the guidance and focus of a strategic plan [14] so it is difficult for CHD leadership to achieve their objectives from work plans drawn from strategic plans that are not informed by evidence from appropriate M\&E. Work plans are management tools as such poor work-planning leads to poor management of the health sector [14]. As the results show, there is generally a weak link between county integrated development plans (CIDP), the work plans and budgets. Work plans do not seem to be guided by CIDPs and the budgets are not executed according to work plans for a number of reasons including poor quality work plans, political interference and inadequate financing for the plans. There is a need for capacity building on annual work-planning and how this is linked with the county strategic plans, as well as strengthening M\&E capabilities of the counties.

In HRH, the key issues are a lack of a structure and strategy for staff attraction, recruitment and retention and a lack of timely performance feedback. These have led to poor job satisfaction and demotivation, and high rates of attrition. Recent studies in Kenya [15] and elsewhere [16, 17] point at 
increased staff demotivation where strategies to attract, recruit and retain staff are lacking. The counties are currently in need of staff attraction, recruitment and retention strategies as well as capacity building on Integrated Human Resource Information System (IHRIS). The Ministry of Health (Kenya) in the current HRH strategy [18] recognizes the challenges in the HRH strategy and is working to lessen their effect on service delivery.

A common problem across most of sub-Saharan Africa and indeed many developing countries is lack of essential supplies to health facilities including drugs, basic equipment and medical supplies [1921]. As demonstrated in this study, lack of essential commodities demotivates staff and highlights not only the incapacity of counties to analyse supply chain data for forecasting and quantification but also a general weakness in the supply chain. It is critical for the devolved units to strengthen their commodity supply systems to ensure constant supplies of essential commodities to improve service delivery.

All the five CHDs demonstrated significant functional capacity in HIS largely because of heavy donor investment in this block. However, there are still some critical gaps that need to be addressed; e.g. although the CHDs have developed many guidelines and other standard operating procedures, these have not been disseminated to the sub-counties and health facilities for quick references. Similar findings have been reported with the observation that such constraints need to be supported by realistic health information strategies and a monitoring system [6]. In addition, improvements in internet access would ease communication and allow staff to update their knowledge and translating to their patients [6].

In the healthcare financing block, the key issues were low funding and weak financial accountability mechanisms. Where funding was available, the allocations were characterized by inefficiency and lack of linkages with evidence-based plans and interventions. Recent reforms such as implementation of PBB to improve efficiency and accountability at the CHDs are important but unfortunately have been unable to effectively reduce wastages and enhance cost-effective interventions. This has been partly blamed on weak PBB skills especially at the sub-county level as well as political interference in health budget allocations. Nevertheless, the health sector generally is underfunded from public domestic sources which stand at $37 \%$ of the total health expenditure [22] against the $60 \%$ benchmark necessary for progress toward UHC[23]. Recent plans to expand enrolment into the National Hospital Insurance Fund (NHIF) as the main avenue to finance UHC is commendable and likely to resolve some of the financing challenges. It is expected that as more households contribute to the NHIF and other health insurance schemes, the national and county governments would be relieved of some financing pressure and reinvest their budgets into health system strengthening. However, efficiency is critical and widely acknowledged that improved efficiency is integral to the aspirations of health systems [24-26] .

Service delivery block is highly linked with the performance of the other blocks. Since the other key blocks- leadership and governance, health financing and $\mathrm{HRH}$, are underperforming, it was expected that service delivery would be grossly affected. However, the high scores in service delivery could be explained on the fact that the data heavily relied on the assessment of key services, namely: HIV/AIDS, malaria, TB, maternal, neonatal and child health services $(\mathrm{MNCH})$ and sanitation services. These services are highly donor-driven and their delivery is not entirely dependent on what happens at the CHDs. Further improvements in service delivery are however, necessary in terms of improved capacity to develop and distribute policies, strategic plans, guidelines and protocols on service delivery. 
On the whole, the evaluation identified critical capacity gaps in the CHDs. Whereas there is need for improvement in all the six building blocks, more attention should be paid to the worst performers: leadership and governance, HRH and financing.

\section{Acknowledgement}

Data for the article were provided by USAID. The article was an analysis of existing data so no funding was sought.

\section{References}

[1] United Nations, UNDG Capacity Assessment Methodology User Guide for national capacity development, United Nations, Geneva, 2008.

[2] OECD, Capacity development: a DAC priority, OECD, 2010.

[3] WHO, Everybody's business - strengthening health systems to improve health outcomes: WHO's framework for action, World Health Organization, Geneva, Switzerland, 2007.

[4] O'Connell, T. et al., "What does universal health coverage mean?," Lancet, 383(9913), 277-9, 2014.

[5] WHO, Domestic resource mobilization for sustainable financing for health in Africa African Region. Working Paper prepared by the WHO Regional Office for Africa, World Health Organization, Geneva, 2017.

[6] Manyazewal, T., "Using the World Health Organization health system building blocks through survey of healthcare professionals to determine the performance of public healthcare facilities," Arch Public Health, 75, 50, 2017.

[7] County Government Turkana, Turkana County Investment Plan - 2016 - 2020, County Government of Turkana, 2016.

[8] Jardfelt, A., Life-saving Cash Transfer Programme Enshrined in Kakamega Law, 2018.

[9] MCS Program, Kenya Country Summary, 2017.

[10] MOH, Differential Maternal Mortality in Kenya: the need to prioritize Interventions, Ministry of Health, Nairobi, 2013.

[11] Hafner, T., and Shiffman, J., "The emergence of global attention to health systems strengthening," Health Policy Plan, 28(1), 41-50, 2013.

[12] Moon, S., "WHO's role in the global health system: what can be learned from global R\&D debates?," Public Health, 128(2), 167-72, 2014.

[13] Swanson, R. C. et al., "Strengthening health systems in low-income countries by enhancing organizational capacities and improving institutions," Global Health, 11,5, 2015.

[14] United Nations, Work-planning for managers, United Nations, Geneva, Switzerland, 2007.

[15] Ministry of State, and IntraHealth, Human Resources for Health (HRH) Assessment in Northern Kenya: An overview of health workforce distribution across 10 counties, Government of Kenya, Nairobi, 2012.

[16] Assefa, F. et al., "Assessment of client satisfaction with health service deliveries at Jimma university teaching hospital," Ethiopian Journal of Health Science 21(2),101-9, 2012.

[17] Engeda, E. H. et al., "Intent to stay in the nursing profession and associated factors among nurses working in Amhara regional state referral hospitals," Ethiopia BMC Nursing., 13(24), 1-8, 2014.

[18] Ministry of Health, Health Sector Human Resources Strategy 2014-2018, Ministry of Health Nairobi, 2014.

[19] Bateman, C., "Drug stock-outs: inept supply-chain management and corruption," S Afr Med J, 103(9), 600-2, 2013.

[20] Cameron, A. et al., "Differences in the availability of medicines for chronic and acute conditions in the public and private sectors of developing countries," Bull World Health Organ, 89(6),41221, 2011.

[21] Wagenaar, B. H. et al., "Stock-outs of essential health products in Mozambique - longitudinal analyses from 2011 to 2013," Trop Med Int Health, 19(7), 791-801, 2014.

[22] Ministry of Health, National Health Accounts 2015/16, Government of Uganda, Kampala, 2017.

[23] McIntyre, D. et al., "What level of domestic government health expenditure should we aspire to for universal health coverage?," Health Econ Policy Law, 12(2), 125-137, 2017.

[24] Farrell, M. J., "The measurement of productive efficiency," Journal of Royal Statistical Society, 120(3), 283-281, 1957. 
International J. of Health Services Research and Policy (2019) 4(1):31 -42

[25] Kirigia, J. M. et al., "Technical efficiency of primary health units in Kailahun and Kenema districts of Sierra Leone," Int Arch Med, 4, 15, 2011.

[26] Zere, E., "Hospital Efficiency in Sub-Saharan Africa: Evidence from South Africa," Research Paper 187, World Institute for Development Economics Research. Helsinki, Finland, 2000. 